\title{
Letramento funcional em saúde de usuários da atenção primária de Altamira, Pará
}

\author{
Functional health literacy in users of family health units from Altamira (state of Pará, Brazil) \\ Alfabetización funcional en salud de usuarios de la atención primaria de Altamira (PA)
}

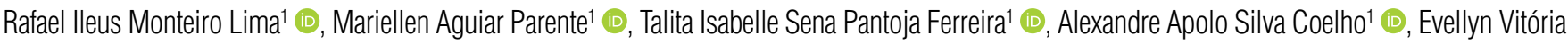

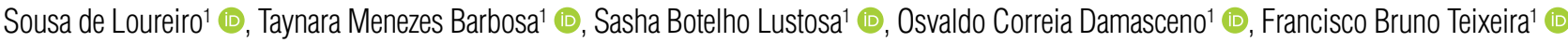

IUniversidade Federal do Pará - Belém (PA), Brasil.

\section{RESUMO}

Introdução: O Letramento Funcional em Saúde (LFS) está associado à capacidade dos pacientes em compreender e utilizar seus conhecimentos em saúde no âmbito do autocuidado e na promoção a saúde. Por isso, torna-se essencial reconhecer o nível de LFS e identificar os fatores que o influenciam. Objetivo: Analisar o nível de letramento funcional em saúde (LFS) de usuários de Unidades de Saúde da Família (USF) na área urbana de Altamira (PA). Métodos: Estudo transversal no qual a coleta de dados ocorreu entre agosto de 2018 e dezembro de 2019 em 12 USF da área urbana. O cálculo amostral foi realizado com base na população urbana de Altamira registrada pelo Censo de 2010. Utilizou-se intervalo de confiança de 95\% com margem de erro de $5 \%$ e prevalência de achados de $50 \%$ em uma população semelhante, encontrando-se assim o valor de 382. Os participantes foram entrevistados a fim de se coletarem informações sobre dados sociodemográficos, e posteriormente foi aplicado um questionário baseado na versão brasileira do Short Test of Functional Health Literacy (S-TOFHLA), instrumento utilizado para a mensuração do LFS. O coeficiente de correlação de Pearson, o teste qui-quadrado, o teste $\mathrm{G}$ e a odds ratio foram utilizados para verificar a relação entre a pontuação do S-TOFHLA e os dados sociodemográficos. Além disso, uma análise de regressão múltipla foi realizada para predizer os fatores que interferem no LFS. Por fim, o teste ANOVA buscou diferenças entre os níveis de LFS dos usuários nas USF analisadas. Resultados: Foram incluídos no estudo 400 participantes, dos quais $59 \%$ apresentaram LFS adequado, $16,5 \%$ limítrofe e $24,5 \%$ inadequado. As variáveis, grau de escolaridade, idade e renda foram associadas ao LFS na população estudada, predizendo, respectivamente, em 46, 26 e $17 \%$ o desempenho no teste S-TOFHLA. Baixa escolaridade e baixa renda aumentam o risco de indivíduos possuírem LFS insatisfatório em cinco e quatro vezes, nessa ordem. Por fim, foram encontradas diferenças entre os níveis de LFS dos usuários das USF analisadas. Conclusões: A população da área urbana de Altamira apresentou alta prevalência de LFS insatisfatório. Sendo assim, no intuito de aumentar os resultados satisfatórios em saúde, os profissionais da região devem adequar as suas formas de comunicação e linguagem às necessidades dos usuários das USF, observando que a adequação da equipe pode trazer melhorias para o entendimento das informações e oportunizar melhores condições de recuperação e autocuidado em saúde.

Palavras-chave: Letramento em saúde. Atenção primária à saúde. Saúde pública. Determinantes sociais da saúde. Educação em saúde.

Como citar: Lima RIM, Parente MA, Ferreira TISP, Coelho AAS, Loureiro EVS, Barbosa TM, Lustosa SB, Damasceno OC, Teixeira FB. Letramento funcional em saúde de usuários da atenção primária de Altamira, Pará. Rev Bras Med Fam Comunidade. 2022;17(44):2763. https://doi.org/10.5712/rbmfc17(44)2763

\author{
Autor correspondente: \\ Francisco Bruno Teixeira \\ E-mail: teixeira.f.bruno@gmail.com \\ Fonte de financiamento: \\ não se aplica \\ Parecer CEP: \\ 2.890.597 \\ Procedência: \\ não encomendado. \\ Avaliação por pares: \\ externa. \\ Recebido em: 24/10/2020. \\ Aprovado em: 06/08/2021. \\ Editor Associado: \\ Leandro David Wenceslau
}




\begin{abstract}
Introduction: Functional Health Literacy $(\mathrm{FHL})$ is associated with patients' ability to understand and use their health knowledge in the context of self-care and health promotion. Therefore, it is essential to recognize the level of FHL and identify the factors that influence it. Objective: To analyze the FHL level in users of Family Health Units (FHU) in the urban area of Altamira (state of Pará, Brazil). Methods: Cross-sectional study. Data collections took place between August 2018 and December 2019 in $12 \mathrm{FHU}$ of the urban area. The sample calculation was based on the urban population of Altamira as recorded by the 2010 Population Census of IBGE. A $95 \%$ confidence interval with a margin of error of $5 \%$ and a prevalence of findings of $50 \%$ in a similar population were used, thus reaching a value of 382 . The participants were interviewed to collect information on sociodemographic data. Subsequently, a questionnaire based on the Brazilian version of the Short Test of Functional Health Literacy (S-TOFHLA) was applied, which is used as an instrument to measure FHL. Pearson's correlation coefficient, Chi-Square test, G-test, and odds ratio were used to verify the correlation between S-TOFHLA score and sociodemographic data. Furthermore, a multiple regression analysis was carried out to predict the factors that affect FHL. Finally, the ANOVA test has sought differences between the users' FHL level in the analyzed family health units. Results: The variables "education level," "age," and "income" were associated with FHL in the studied population, and predicted, respectively, 46, 26, and $17 \%$ of the performance in the S-TOFHLA test. Low education level and low income increase the risk of individuals having unsatisfactory FHL by five and four times, respectively. Finally, differences in FHL between users of the analyzed FHU were found. Conclusions: The population of the urban area of Altamira presented a high prevalence of unsatisfactory FHL. Therefore, seeking to increase the positive health results, healthcare professionals of the region must adapt their ways of communication and language to the needs of FHU users, observing that the team adequacy can improve the understanding of information and provide better conditions of recovery and self-care.
\end{abstract}

Keywords: Health literacy. Primary health care. Public health. Social determinants of health. Health education.

\title{
RESUMEN
}

Introducción: La de Alfabetización Funcional en Salud (AFS) se asocia con la capacidad de los pacientes para comprender y utilizar sus conocimientos sobre salud en el contexto del autocuidado y la promoción de la salud. Por lo tanto, se vuelve fundamental reconocer el nivel de LFS e identificar los factores que influyen en él. Objetivo: Analizar el nivel de alfabetización funcional en salud (AFS) de usuarios de Unidades Salud de la Familia (USF) en el área urbana de Altamira (PA). Métodos: Estudio transversal en el cual la recolección de datos se realizó entre agosto del 2018 y diciembre del 2019 en 12 USF del área urbana. El cálculo de la muestra se realizó a partir de la población urbana de Altamira registrada por el Censo de 2010. Se utilizó intervalo de confianza del 95\% con un margen de error del $5 \%$ y una prevalencia de hallazgos del $50 \%$ en una población semejante, encontrando así el valor de 382. Los participantes fueron entrevistados a fin de recopilar informaciones sobre datos sociodemográficos, y posteriormente se aplicó un cuestionario basado en la versión brasileña del Short Test of Functional Health Literacy (S-TOFHLA), que es un instrumento utilizado para medir el AFS. Se utilizó el coeficiente de correlación de Pearson, la prueba de Chi-Cuadrado, la prueba $\mathrm{G}$ y los odds ratios fueron utilizados para verificar la relación entre la puntuación del S-TOFHLA y los datos sociodemográficos. Además, se realizó un análisis de regresión múltiple para predecir los factores que afectan el AFS. Finalmente, la prueba ANOVA buscó diferencias entre el nivel de ASF de los usuarios en las USF analizadas. Resultados: Se incluyeron 400 participantes en el estudio, de los cuales el $59 \%$ tenía un AFS adecuado, $16,5 \%$ limítrofe y el $24,5 \%$ inadecuado. Las variables nivel educativo, la edad y los recursos se asociaron con el AFS en la población estudiada, y predicen el $46 \%, 26 \%$ y el $17 \%$ del rendimiento en la prueba S-TOFHLA, respectivamente. La baja escolaridad y los bajos recursos aumentan el riesgo de que las personas tengan AFS insatisfactorio 5 y 4 veces, en ese orden. Finalmente, se encontraron diferencias entre los niveles de AFS de los usuarios de las USF analizadas. Conclusiones: La población del área urbana de Altamira mostró una alta prevalencia de AFS insatisfactorio. Entonces, para aumentar los resultados satisfactorios en salud, los profesionales de la región deben adecuar sus formas de comunicación y lenguaje a las necesidades de los usuarios de las USF, señalando que la educación del equipo puede aportar mejoras a la comprensión de la información y proporcionar mejores condiciones de recuperación y autocuidado en salud.

Palabras-clave: Alfabetización en salud. Atención primaria de salud. Salud pública. Determinantes sociales de la salud. Educación en salud.

\section{INTRODUÇÃO}

Letramento funcional em saúde (LFS) refere-se ao conhecimento, à motivação e à competência dos pacientes para acessar, compreender, avaliar e aplicar informação em saúde, de forma a realizar julgamentos e tomar decisões cotidianas no que tange ao autocuidado, à prevenção de doenças e à promoção da saúde, buscando manter ou melhorar a qualidade de vida. ${ }^{1,2}$ Nessa perspectiva, as pessoas tornam-se protagonistas das informações em saúde em todos os contextos de interação social. ${ }^{1,3}$

$O$ acesso às informações em saúde é significativo e crucial para garantir que as pessoas alcancem seu potencial máximo de saúde. Nesse contexto, encontram-se os papéis da educação e do adequado LFS. ${ }^{1,2}$ A Organização Mundial da Saúde ${ }^{4}$ identificou o letramento como um dos determinantes sociais da 
saúde por ser elemento importante na estratégia de redução da desigualdade. A avaliação e a determinação dos níveis de LFS são fundamentais por permitir, no sistema de saúde, o desenvolvimento de maneiras adequadas de transmitir informações aos indivíduos com habilidades limitadas de compreensão de leitura e numeramento. ${ }^{5}$

O LFS inadequado está associado a erros e à baixa adesão ao tratamento, ${ }^{6-8}$ à maior frequência de internações, ao controle desfavorável sobre doenças de maior risco de morbimortalidade ${ }^{9-11}$ e à diminuição do rastreio e da qualidade de vida em pacientes com câncer. ${ }^{12}$ Logo, no contexto atual, no qual dependemos da participação de todos para a contenção de doenças infectocontagiosas e a disseminação de informações equivocadas, o LFS torna-se essencial para o sucesso de políticas públicas implementadas em nível local, nacional e/ou global. ${ }^{1-3}$

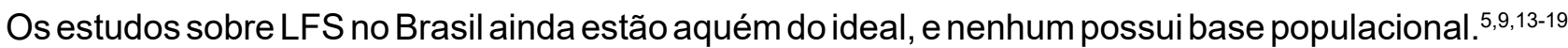
Em relação à região Norte, que possui peculiaridades em comparação às outras regiões do país, observamos apenas um estudo sobre a temática. ${ }^{15}$

Nesse cenário de particularidades regionais, encontra-se Altamira, município localizado na mesorregião do Xingu, que desde 2011 sofre os impactos relacionados à construção da usina hidrelétrica (UHE) Belo Monte, maior hidrelétrica inteiramente brasileira. O empreendimento aumentou o fluxo migratório no município, o que ocasionou uma explosão populacional não planejada que se refletiu diretamente nos serviços de saúde e na economia local. ${ }^{20,21}$

Diante disso, mensurar o LFS nesse município é essencial para identificar as dificuldades dos indivíduos e das comunidades no acesso, na compreensão e no uso das informações em saúde, de maneira a viabilizar propostas de intervenções adequadas a essas necessidades. Assim, o objetivo do estudo foi analisar o nível de LFS de usuários da atenção primária na área urbana do município de Altamira.

\section{MÉTODOS}

\section{Tipo de estudo e aspectos ético-legais}

Tratou-se de um estudo transversal, de caráter quantitativo, conduzido em Unidades de Saúde da Família (USF) da região urbana de Altamira, no Pará. A pesquisa foi aprovada pelo Comitê de Ética em Pesquisa em Seres Humanos do Núcleo de Medicina Tropical da Universidade Federal do Pará, sob número de parecer 2.890.597. Os participantes receberam esclarecimentos sobre os objetivos do estudo, o caráter opcional de sua participação e a necessidade de assinar o Termo de Consentimento Livre e Esclarecido, como preconizado pelas Resoluções 466/2012 e 510/2016 do Conselho Nacional de Saúde. Além disso, obteve-se a permissão dos autores que elaboraram a versão brasileira do teste adotado para a avaliação do LFS.

\section{Local do estudo}

O estudo foi conduzido em 12 USF da região urbana de Altamira (PA). O município é referência para toda região do Xingu e possui população estimada em 2019 de 114.594 habitantes, dos quais aproximadamente 75\% habitam na área urbana, segundo Censo de 2010 (85.945). ${ }^{22}$ A localidade compõe, com Anapu, Brasil Novo, Medicilândia, Pacajá, Placas, Porto de Moz, Senador José Porfírio, Uruará e 
Vitória do Xingu, o $10^{\circ}$ Centro Regional de Saúde/Secretaria Estadual de Saúde do Pará (CRS/SESPA). Além disso, é fortemente marcada pela presença de povos indígenas e comunidades extrativistas.

A escolha das USF deu-se por amostra de conveniência, excluindo-se seis USF do município. Os critérios para as escolhas basearam-se em orientações da Secretaria Municipal de Saúde, que, por sua vez, indicou as USF com maior fluxo de atendimento no período de coleta de dados do estudo. Para isso, houve análise das agendas médicas, na qual foi verificado o número de usuários que haviam consultado as unidades nos três meses anteriores. Por questões éticas, neste artigo, as USF serão enumeradas de 1 a 12 para identificação.

\section{População e amostra}

O cálculo amostral foi realizado com base na população urbana de Altamira registrada pelo Censo de 2010. Utilizou-se intervalo de confiança de $95 \%$, com margem de erro de $5 \%$, e prevalência de achados de $50 \%$ em uma população semelhante. Verificou-se que a amostra dessa pesquisa, assumindo-se os critérios acima estabelecidos, deveria ser de no mínimo 383 indivíduos.

Os critérios de inclusão do estudo foram:

1. Ser usuário de USF da região urbana de Altamira;

2. Possuir idade igual ou superior a 18 anos; e

3. Ser alfabetizado.

Os critérios de exclusão foram:

1. Ser portador de doença neurodegenerativa ou distúrbio psiquiátrico; ou

2. Estar sob tratamento com medicação que comprometa a cognição.

Para a seleção dos participantes, foi convidado a participar o último usuário na fila de espera para o atendimento, e, caso ele não aceitasse, era convidado o usuário imediatamente anterior. Esse critério possuiu como base o tempo necessário para que o participante pudesse preencher os questionários propostos sem interrupção, o que inviabilizaria a análise.

No fim das coletas, participaram do estudo 400 indivíduos distribuídos entre as 12 USF. As USF com maior número de atendimentos repassados pela Secretaria Municipal de Saúde possuíram 40 participantes inclusos no estudo (USF 1, USF 3, USF 5 e USF 12), enquanto as demais contribuíram com 30 usuários cada.

\section{Coleta de dados e instrumentos}

A coleta de dados ocorreu entre agosto de 2018 e dezembro de 2019, em 12 USF da região urbana, por meio da aplicação de dois instrumentos. O primeiro era aplicado após a abordagem inicial dos participantes, que tinha a finalidade de coleta de informações sociodemográficas. As variáveis, sexo, etnia, idade, grau de escolaridade e renda familiar bruta dos participantes foram coletadas.

O segundo instrumento avaliou o LFS, adotando-se a versão brasileira do Short Test of Functional Health Literacy (S-TOFHLA), traduzido e adaptado culturalmente por Carthery-Goulart et al. ${ }^{5} \mathrm{O}$ teste é composto de duas etapas: a primeira analisa a compreensão textual e a leitura; a segunda avalia a capacidade de atuar com números. 
O questionário de análise do LFS da primeira etapa consistiu em duas passagens: passagem $A$, que se referia ao preparo para o exame de raio $X$ do trato gastrointestinal superior; e passagem $B$, relacionada aos direitos e responsabilidades dos pacientes em relação ao serviço de saúde. Cada passagem possuía, a cada sexto ou sétimo termo, um espaço em branco no qual o entrevistado deveria selecionar, de uma lista de quatro palavras, a que melhor completasse a frase. Havia 36 lacunas e a pontuação total dos textos de compreensão de leitura era de 72 pontos, isto é, uma lacuna corretamente concluída marcava dois pontos. ${ }^{5}$

O teste para análise do letramento quantitativo em saúde (numeramento) compreendeu quatro cartões com informações sobre ingestão de medicamentos, data de consulta e resultado de teste laboratorial. Cada cartão foi entregue separadamente e, em seguida, o pesquisador realizava uma pergunta relacionada ao respectivo item. A etapa de numeramento totalizava 28 pontos e, para cada acerto, foram atribuídos sete pontos. ${ }^{5}$

A pontuação total do teste era de 100, e a classificação do nível de letramento deu-se conforme o escore obtido: 5

- $\quad$ LFS inadequado (escore 0-53);

- $\quad$ LFS marginal ou limítrofe (escore 54-66);

- $\quad$ LFS adequado (escore 67-100).

A etapa de compreensão de leitura deveria ser executada em $7 \mathrm{~min}$, enquanto o tempo máximo para a etapa de numeramento era de $5 \mathrm{~min}$. Os itens concluídos após o participante ultrapassar esses períodos não foram analisados.

\section{Análise estatística}

Estatísticas descritivas foram usadas para resumir informações quantitativas sobre a amostra geral. Inicialmente, foi realizado o teste do qui-quadrado ou o teste $\mathrm{G}$ de independência (quando os pré-requisitos do teste anterior não eram atendidos) para averiguar a associação entre as variáveis sociodemográficas categóricas ou ordinais e a classificação do nível de LFS. Para a variável sociodemográfica contínua (idade), o coeficiente de correlação de Pearson verificou a sua relação com a pontuação do S-TOFHLA. Em seguida, as variáveis que apresentaram associação na amostra geral foram submetidas ao teste de regressão linear múltipla, possuindo como variável dependente o escore obtido no teste de letramento (0-100).

O odds ratio foi usado para averiguar o grau de risco entre as variáveis que apresentaram associação com o desempenho no S-TOFHLA. Nessa análise, dados os pré-requisitos do teste estatístico, a variável dependente foi dicotomizada em:

1. LFS satisfatório para os participantes que obtiveram a classificação "adequada"; e

2. LFS insatisfatório para os participantes que possuíram a classificação "inadequada" ou "marginal".

As variáveis, grau de escolaridade e renda também foram dicotomizadas, sendo classificadas como:

1. Escolaridade satisfatória - participantes com ensino médio concluído ou ensino superior completo ou incompleto; e

2. Escolaridade insatisfatória - participantes que não concluíram o ensino médio; e

3. Participantes com renda familiar superior a dois salários-mínimos; e

4. Participantes com renda familiar igual ou inferior a dois salários-mínimos. 
As variáveis idade e etnia não foram dicotomizadas neste estudo, mesmo quando possuíam associação com os escores do S-TOFHLA.

Por fim, buscando verificar a diferença nos escores do S-TOFHLA entre as USF analisadas, os dados foram submetidos à análise de variância (ANOVA) de uma via, seguida do pós-teste de Tukey quando diferenças significativas foram detectadas. Os resultados de variáveis contínuas foram expressos em médiaterro padrão, e para todos os testes foi adotado valor de $p \leq 0,05$ como indicativo de diferença estatística significante.

\section{RESULTADOS}

O estudo obteve a participação de 400 indivíduos divididos em 12 USF da região urbana de Altamira, com média de idade de 33,5 e desvio padrão de 11,85. A amostra foi composta, majoritariamente, de indivíduos do sexo feminino $(79,5 \%)$, pardos $(67,25 \%)$, com ensino médio completo $(29,75 \%)$ e com renda familiar bruta declarada de um a dois salários-mínimos (27,25\%). A análise sociodemográfica mostrou dados preocupantes com relação aos usuários das USF de Altamira. Constatou-se a pouca procura de atendimentos por parte do sexo masculino (20,5\%), uma considerável parcela de indivíduos sem o ensino médio completo (16\%) e percentual igualmente importante de entrevistados (16\%) com renda familiar inferior a um salário mínimo (Tabela 1).

Tabela 1. Distribuição das características sociodemográficas dos participantes em associação com o nível do letramento funcional em saúde.

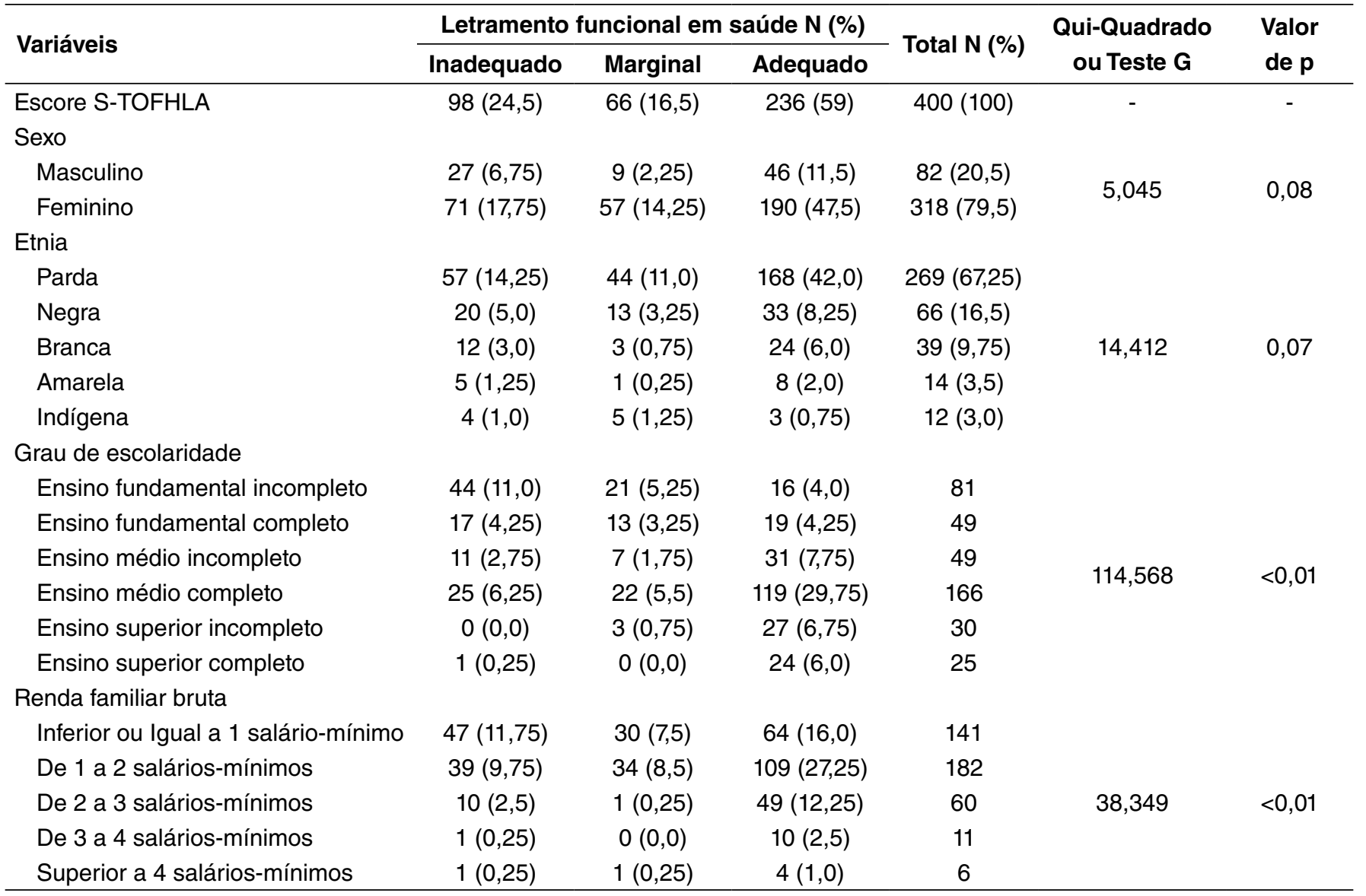

S-TOFHLA: Short Test of Functional Health Literacy 
A pesquisa revelou a média do escore do S-TOFHLA de $70,97 \pm 1,13$, além do que mais da metade dessa amostra apresentou adequado nível de LFS (59\%). No entanto, ao se considerar que $16,5 \%$ atingiram letramento limítrofe e $24,5 \%$ apresentaram nível inadequado, certificou-se que a cada dez pacientes atendidos por profissionais de saúde em uma USF de Altamira quatro poderiam sair da consulta com dúvidas ou equívocos em relação ao seu processo saúde-doença (Tabela 1).

As associações entre as variáveis sociodemográficas categóricas ou ordinais com o nível de LFS foram avaliadas utilizando o teste do qui-quadrado para a variável sexo. Para as demais variáveis, utilizou-se o teste $G$ de independência, pois elas apresentaram frequência esperada inferior a 5 em um dos grupos e foram organizadas em tabelas de contingência $(/ \times c)$, sendo pelo menos I ou c diferente de 2 (Tabela 1). Essa análise demonstrou que o grau de escolaridade $\left(\chi^{2}=114.568, p<0,01\right)$ e a renda familiar $\left(\chi^{2}=38.349, p<0,01\right)$ são associados com o LFS, no entanto as variáveis sexo $\left(\chi^{2}=5.045, p=0,08\right)$ e etnia $\left(\chi^{2}=14.4120, p=0,07\right)$ não demonstram associação (Tabela 1).

Para a variável idade, o coeficiente de correlação de Pearson demonstrou associação com o escore do LFS $(r=-0,33 ; p<0,01)$. Assim, as variáveis idade, grau de escolaridade e renda familiar bruta foram submetidas à análise de regressão linear múltipla para averiguar suas participações como preditoras do escore do S-TOFHLA aplicado. A análise resultou em um modelo estatisticamente significativo $\left[F(1,395)=16,296 ; p<0,01 ; R^{2}=0,391\right]$ e mostrou que o grau de escolaridade $(\beta=0,46 ; t=10,794 ; p<0,01)$, a idade $(\beta=-0,26 ; t=6,495 ; p<0,01)$ e a renda familiar $(\beta=0,17 ; t=4,037 ; p<0,01)$ são preditoras do LFS; ou seja, 46, 26 e 17\% do desempenho dos usuários no teste S-TOFHLA são explicados pelas variáveis grau de escolaridade, idade e renda, respectivamente, devendo outros fatores não analisados neste estudo atuarem como preditores de $11 \%$ do desempenho.

Diante desses resultados, o odds ratio avaliou o grau de risco entre as variáveis escolaridade e renda com a classificação de desempenho no S-TOFHLA. Nessa análise as variáveis foram dicotomizadas, conforme descrito na seção anterior. Assim, observou-se que a probabilidade de um indivíduo que concluiu o ensino médio possuir adequado LFS é cerca de cinco vezes maior que a dos que não concluíram. De modo semelhante, a probabilidade de indivíduos que têm renda familiar superior a dois saláriosmínimos possuírem adequado LFS é quase quatro vezes maior que a dos que têm renda inferior ou igual a dois salários-mínimos (Tabela 2 ).

Tabela 2. Distribuição das características sociodemográficas dos participantes e risco em relação com o nível do letramento funcional em saúde.

\begin{tabular}{|c|c|c|c|c|}
\hline \multirow[b]{2}{*}{ Variáveis } & \multicolumn{2}{|c|}{ Letramento funcional em saúde $\mathrm{N}(\%)$} & \multirow{2}{*}{$\begin{array}{l}\text { Odds } \\
\text { ratio }\end{array}$} & \multirow{2}{*}{$\begin{array}{l}\text { Intervalo de } \\
\text { confiança }\end{array}$} \\
\hline & $\begin{array}{l}\text { Letramento } \\
\text { satisfatório }\end{array}$ & $\begin{array}{c}\text { Letramento } \\
\text { insatisfatório }\end{array}$ & & \\
\hline \multicolumn{5}{|l|}{ Grau de escolaridade } \\
\hline Escolaridade adequada & $170(42,5)$ & $51(12,75)$ & \multirow{2}{*}{5,707} & \multirow{2}{*}{$3,690<\mu<8,826$} \\
\hline Escolaridade inadequada & $66(16,5)$ & $113(28,25)$ & & \\
\hline \multicolumn{5}{|l|}{ Renda familiar bruta } \\
\hline Renda superior a dois salários-mínimos & $63(15,75)$ & $14(3,5)$ & \multirow{2}{*}{3,902} & \multirow{2}{*}{$2,101<\mu<7,246$} \\
\hline Renda inferior ou igual a dois salários-mínimos & $173(43,25)$ & $150(37,5)$ & & \\
\hline
\end{tabular}


A Tabela 3 resume as informações quanto ao escore obtido pelo S-TOFHLA e a diferença entre as USF analisadas conforme o teste ANOVA ( $F=4.464 ; p<0,01)$. O pós-teste de Tukey revelou que os usuários que frequentam a USF 12 possuem LFS inferior a quatro USF de Altamira. Além disso, verificouse que os usuários que são adscritos na USF 1 apresentam o melhor nível de LFS, possuindo diferença estatística em relação a três USF.

Tabela 3. Comparação dos escores do Short Test of Functional Health Literacy entre as Unidades de Saúde da Família da área urbana de Altamira.

\begin{tabular}{lccc}
\hline USF & $\begin{array}{c}\text { Média } \pm \text { erro padrão do escore } \\
\text { S-TOFHLA }\end{array}$ & Diferente em relação as USF & $\begin{array}{c}\text { Valor de p (ANOVA com } \\
\text { pós-teste de Tukey) }\end{array}$ \\
\hline USF 1 & USF 12 & $<0,01$ \\
& $82,81 \pm 2,65$ & USF 11 & $=0,01$ \\
USF 2 & USF 10 & $=0,03$ \\
USF 3 & $79,07 \pm 3,616$ & USF 12 & $<0,01$ \\
USF 4 & $78,35 \pm 3,088$ & USF 12 & $<0,01$ \\
USF 5 & $74,57 \pm 3,776$ & USF 12 & $=0,02$ \\
USF 6 & $72,68 \pm 3,623$ & USF 12 & $=0,03$ \\
USF 7 & $70,77 \pm 3,758$ & - & - \\
USF 8 & $68,83 \pm 4,669$ & - & - \\
USF 9 & $68,80 \pm 3,749$ & - & - \\
USF 10 & $68,76 \pm 4,686$ & - & - \\
USF 11 & $64,93 \pm 3,872$ & - & - \\
& $63,63 \pm 4,395$ & - & - \\
& & USF 1 & $<0,01$ \\
USF 12 & USF 2 & $<0,01$ \\
& & USF 3 & $<0,01$ \\
Amostra total & USF 4 & $=0,02$ \\
\hline S-TOFHLA: & USF 5 & $=0,03$ \\
& $56,19 \pm 3,597$ & - & - \\
& & &
\end{tabular}

S-TOFHLA: Short Test of Functional Health Literacy; USF: Unidades de Saúde da Família

\section{DISCUSSÃO}

Este estudo mostra, pela primeira vez, uma análise sobre o LFS de uma população que utiliza serviços públicos de saúde em um município do interior da região Norte do Brasil. Altamira sofreu vários impactos sociais, econômicos e demográficos após a instalação da UHE Belo Monte, pois não estava preparado para o intenso fluxo migratório no período de implantação do empreendimento, de modo que diversos problemas ficaram como herança. ${ }^{20,21}$ Nesse contexto, é essencial conhecer suas características populacionais para oferecer serviços e atividades de educação em saúde adequados aos seus habitantes. Além disso, esta pesquisa ressalta que, após grandes mudanças sociodemográficas em uma região, fazse necessário reconhecer o novo cenário implementado.

A fim de desenvolver instrumentos de avaliação do LFS, vários testes foram elaborados em diferentes países, com destaque para o Test of Functional Health Literacy in Adults (TOFHLA), que classifica o grau 
de LFS com base na avaliação da compreensão de leitura e da habilidade numérica. ${ }^{23}$ Para reduzir o tempo de aplicação, desenvolveu-se uma versão abreviada do teste que foi adaptada para o português por Carthery-Goulart et al. ${ }^{5}$ Os autores classificaram o teste como ferramenta adequada de avaliação do LFS na população brasileira, por ser de fácil aplicação e adaptável ao contexto do Sistema Único de Saúde (SUS).

Nossos resultados indicaram que $40 \%$ dos usuários de USF de Altamira apresentaram LFS insatisfatório (inadequado ou marginal), desfecho pouco superior ao encontrado em hospitais de São Paulo. ${ }^{5}$ Em comparação com pesquisas de outros países, a prevalência de alfabetização limitada em saúde é de $26 \%$ da população nos Estados Unidos ${ }^{24}$ e entre 29 e $62 \%$ das populações de oito países europeus. ${ }^{25}$ Todavia, esses estudos utilizaram instrumentos de pesquisa diferentes para a mensuração do LFS. Diante disso, o município de Altamira exibe uma parcela considerável da população com baixo índice de LFS.

Além disso, esta pesquisa revelou que idade, grau de escolaridade e renda são fatores preditivos para o LFS na população analisada, fato evidenciado pelas análises de associação/correlação e de regressão. Alguns estudos brasileiros corroboram a relação do primeiro fator. ${ }^{9,13,19}$ Essa constatação é justificada na literatura, pois os idosos são propícios a possuir baixo nível de LFS em razão do envelhecimento e da vulnerabilidade social. , 26

A associação de LFS e grau de escolaridade foi observada neste estudo, assim como em outros realizados em diferentes regiões do Brasil.5,9,13,15,16,18,19 Nesse contexto, a escolaridade configura-se como o principal fator preditivo para o nível de LFS. Entretanto, vale ressaltar que dados mostram que mais de $12 \%$ dos usuários possuem escolaridade adequada e também LFS insatisfatório. Esse fato destaca que os anos de escolaridade sozinhos podem não ser um indicador confiável de habilidades de compreensão de leitura, pois o letramento exige contextos em saúde que são frequentemente mais complexos do que aqueles da vida cotidiana. ${ }^{27}$ Assim, é necessária também a identificação de outros fatores que possam prever esse baixo LFS, como fatores socioeconômicos, hábitos de leitura e escrita e a prevalência de analfabetismo funcional. ${ }^{5}$

A definição da Organização das Nações Unidas para a Educação, a Ciência e a Cultura (UNESCO) de analfabetismo funcional inclui a pessoa que, mesmo sabendo ler e escrever ou fazer cálculos básicos, é incapaz de interpretar a aplicação dessas habilidades no cotidiano. ${ }^{28}$ Embora pesquisas mostrem melhorias nos níveis de alfabetismo no Brasil ao longo dos últimos anos, em 2018 o índice de analfabetos funcionais ainda permanecia elevado, com 29\% entre os indivíduos de 15 a 64 anos, de acordo com o Indicador de Alfabetismo Funcional (INAF). ${ }^{29}$ Esse dado assemelha-se à porcentagem de entrevistados em nosso estudo - considerando-se apenas os indivíduos com escolaridade adequada, 23\% tiveram letramento insatisfatório.

No entanto, ressalta-se ainda a importância da conclusão do ensino médio, visto que a probabilidade de obter LFS adequado para um indivíduo que concluiu esse grau é cerca de cinco vezes maior que a dos que não concluíram. Diante disso, medidas para a elevação dos níveis do LFS devem ser direcionadas tanto para o incentivo à conclusão do ensino básico quanto para a melhoria da capacidade de interpretação do contexto socioeconômico que esses indivíduos vivenciam.

Dados do INAF no ano de 2011 mostraram que 36\% das pessoas pertencentes a famílias com renda entre um e dois salários-mínimos estão classificadas no nível de analfabetismo funcional. ${ }^{29}$ Comparando-se esse dado com os da nossa pesquisa, analisando apenas participantes com renda familiar bruta inferior ou igual a dois salários-mínimos, verificou-se que esses indivíduos têm probabilidade de LFS inadequado quatro vezes maior que a dos que possuem renda superior a essa faixa. Além disso, 
quase metade dos participantes com renda baixa também possui LFS inadequado. Esse quadro remete à influência do nível socioeconômico no LFS, pois o letramento não depende exclusivamente de características e habilidades individuais, mas também do seu contexto sociocultural. ${ }^{1,30}$ Logo, as características socioeconômicas refletirão nas condições de moradia, alimentação, trabalho, acesso à saúde e educação de qualidade. ${ }^{31}$ Por conseguinte, para reduzir as desigualdades, é necessária a combinação de políticas sociais, investimentos em infraestrutura, melhoria na economia, no mercado de trabalho e na remuneração, principalmente dos mais pobres.

Assim, este estudo definiu que grau de escolaridade, idade e renda prognosticam o desempenho dos usuários de USF de Altamira no teste S-TOFHLA, em 46, 26 e 17\%, respectivamente (Tabela 2). Com base nisso, o LFS pode ser aprimorado mediante acesso a melhores condições socioeconômicas, à educação e à estimulação da interpretação do cotidiano. Melhorar o LFS envolve tanto a transmissão de informações em saúde quanto o estímulo ao protagonismo no processo saúde-doença, objetivo alcançado por meio de formas de comunicação eficientes e de ações educativas baseadas em cada comunidade. ${ }^{3}$

Nesse contexto de ações individualizadas em diferentes localidades, faz-se possível a comparação dos escores do S-TOFHLA entre as USF da área urbana de Altamira (Tabela 3). A Tabela indica que os usuários da USF 12 apresentaram menor nível de LFS entre as USF, significativamente menor em comparação a quatro delas. Pode-se relacionar o seu menor escore com a situação socioeconômica e cultural da população assistida por essa unidade. AUSF 12 localiza-se em um dos reassentamentos urbanos coletivos (RUC) do município, construídos para abrigar as famílias das áreas alagadas pela construção da UHE Belo Monte - ou seja, uma das populações mais pobres e marginalizadas do município ${ }^{20,21}$ e que, portanto, tem menores oportunidades de estudo, o que se reflete diretamente no seu nível de LFS e nas suas condições de saúde. ${ }^{31}$ Assim, o reconhecimento dessa fragilidade é extremamente útil para adequar os serviços e os atendimentos da USF 12 às necessidades dessa população. Desse modo, é crucial reconhecer as características individuais dos usuários de diferentes USF em um mesmo território, identificando suas fragilidades e particularidades para que sejam realizadas intervenções adequadas. ${ }^{3}$

O LFS, além de instrumento atuante no processo saúde-doença, age como ferramenta essencial para a efetivação da participação social nas políticas públicas do SUS. Isso porque propicia ao usuário o direito à informação sobre o autocuidado e os serviços de saúde, para que ele participe das decisões que envolvam as políticas públicas e exerça o seu papel de fiscalizador do sistema. ${ }^{1,2,16}$

Quando uma sociedade permite a criação e a acessibilidade de informações adequadas às necessidades informacionais das pessoas, a assimilação do conhecimento transmitido proporciona a compreensão e a aplicação produtiva das decisões individuais e sociais. ${ }^{30}$ Nessa circunstância, tornase necessário um esforço crescente no ajuste dos sistemas de saúde ao LFS da população, de modo a identificar e adequar o acesso a serviços e informações para populações vulneráveis. ${ }^{23}$

\section{CONCLUSÃO}

Este estudo mostrou que a população da área urbana de Altamira apresentou alta prevalência de LFS inadequado. Diante disso, no intuito de aumentar os resultados positivos em saúde, os profissionais de saúde da região devem adequar as suas formas de comunicação e linguagem às necessidades dos usuários das USF, pois isso traz melhorias para o entendimento das informações e oportuniza melhores condições de recuperação e autocuidado. Nesse cenário, é importante a realização de pesquisas com esse viés, que possam diagnosticar os usuários de USF isoladamente no que se refere ao LFS. 
Este estudo possui a limitação de não conseguir abranger todas as USF urbanas do município, além de não realizar uma avaliação regionalizada em cada área adscrita. Essa análise poderia fornecer informações mais específicas dos territórios, como por exemplo das variáveis que mais influenciam o LFS na população coberta por cada equipe da Estratégia Saúde da Família. No entanto, é notória sua importância, visto que abre possibilidades ao sugerir novas pesquisas na temática do LFS, tanto em pequenas áreas como em âmbito populacional no município e em outras localidades.

Assim, com os dados deste estudo e de outros que possam a vir somar informações, será possível aperfeiçoar a atenção primária no âmbito do SUS e de outros sistemas de saúde, mediante estratégias que ofereçam subsídios e ferramentas adequadas para diminuir as disparidades em saúde atribuídas ao baixo nível de LFS.

\section{CONFLITO DE INTERESSES}

Nada a declarar.

\section{CONTRIBUIÇÕES DOS AUTORES}

RIML: Escrita - primeira redação, Investigação, Metodologia. MAP: Escrita - primeira redação, Investigação, Metodologia. TISPF: Escrita - primeira redação, Investigação, Metodologia. AASC: Escrita primeira redação, Investigação, Metodologia. EVSL: Escrita - primeira redação, Investigação, Metodologia. TMB: Escrita - primeira redação, Investigação, Metodologia. SBL: Escrita - primeira redação, Investigação, Metodologia. OCD: Conceituação, Escrita - revisão e edição, Supervisão, Recursos. FBT: Administração do projeto, Conceituação, Escrita - revisão e edição, Curadoria de dados, Supervisão, Recursos.

\section{REFERÊNCIAS BIBLIOGRÁFICAS}

1. Sørensen K, Van den Broucke S, Fullam J, Doyle G, Pelikan J, Slonska Z, et al. Health literacy and public health: a systematic review and integration of definitions and models. BMC Public Health. 2012;12:80. https://doi.org/10.1186/1471-2458-12-80

2. Parker RM, Ratzan S. Re-enforce, not re-define health literacy-moving forward with Health Literacy 2.0. J Health Commun. 2019;24(12):923-5. https://doi.org/10.1080/10810730.2019.1691292

3. Nutbeam D. Defining, measuring and improving health literacy. Health Evaluation and Promotion. 2015;42(4):450-55. http:// doi.org/10.7143/jhep.42.450

4. Wilkinson R, Marmot M. Social determinants of health: the solid facts. $2^{\text {nd }}$ ed. Geneva: World Health Organization; 2004. Disponível em: https://www.euro.who.int/_data/assets/pdf_file/0005/98438/e81384.pdf

5. Carthery-Goulart MT, Anghinah R, Areza-Fegyveres R, Bahia VS, Brucki SMD, Damin A, et al. Performance of a Brazilian population on the test of functional health literacy in adults. Rev Saude Publica. 2009;43(4):631-8. https://doi.org/10.1590/ s0034-89102009005000031

6. Thurston MM, Bourg CA, Philips BB, Huston SA. Impact of health literacy level on aspects of medication nonadherence reported by underserved patients with type 2 diabetes. Diabetes Technol. Ther. 2015;17(3):187-93. https://doi.org/10.1089/ dia.2014.0220

7. Muellers KA, Chen L, O'Conor R, Wolf MS, Federman AD, Wisnivesky JP. Health literacy and medication adherence in COPD patients: when caregiver presence is not sufficient. COPD. 2019;16(5-6):362-7. https://doi.org/10.1080/15412555.20 19.1665007

8. Skoumalova I, Kolarcik P, Geckova AM, Rosenberger J, Majernikova, M, Klein D, et al. Is health literacy of dialyzed patients related to their adherence to dietary and fluid intake recommendations? Int J Environ Res Public Health. 2019;16(21):4295. https://doi.org/10.3390/ijerph16214295

9. Sampaio HAC, Carioca AAF, Sabry MOD, Santos PM, Coelho MAM, Passamai MPB. Letramento em saúde de diabéticos tipo 2: fatores associados e controle glicêmico. Ciênc Saúde Coletiva. 2015;20(3):865-74. https://doi.org/ 10.1590/141381232015203.12392014

10. Lindahl B, Norberg M, Johansson $\mathrm{H}$, Lindvall $\mathrm{K}, \mathrm{Ng} \mathrm{N}$, Nordin $\mathrm{M}$, et al. Health literacy is independently and inversely associated with carotid artery plaques and cardiovascular risk. Eur J Prev Cardiol. 2020;27(2):209-15. https://doi. org/10.1177/2047487319882821 
11. Rafferty AP, Winterbauer NL, Luo H, Bell RA, Little NRG. Diabetes self-care and clinical care among adults with low health literacy. J Public Health Manag Pract. 2021;27(2):144-53. https://doi.org/10.1097/PHH.0000000000001050

12. Nilsen ML, Moskovitz J, Lyu L, Harrison C, Randazza E, Peddada S, et al. Health literacy: impact on quality of life in head and neck cancer survivors. Laryngoscope. 2020;130(10):2354-9. https://doi.org/10.1002/lary.28360

13. Apolinário D, Braga RCOP, Magaldi RM, Busse AL, Campora F, Brucki S, et al. Short assessment of health literacy for portuguese-speaking adults. Rev Saude Publica. 2012;46(4):702-11. https://doi.org/10.1590/S003489102012005000047

14. Souza JG, Apolinario D, Magaldi RM, Busse AL, Campora F, Jacob-Filho W. Functional health literacy and glycaemic control in older adults with type 2 diabetes: a cross-sectional study. BMJ Open. 2014;4(2):e004180. https://doi.org/10.1136/ bmjopen-2013-004180

15. Santos MIPO, Portella MR. Conditions of functional health literacy of an elderly diabetics group. Rev Bras Enferm. 2016;69(1):144-52. https://doi.org/10.1590/0034-7167.2016690121i

16. Moraes KL, Brasil VV, Oliveira GF, Cordeiro JABL, Silva AMTC, Boaventura RP, et al. Functional health literacy and knowledge of renal patients on pre-dialytic treatment. Rev Bras Enferm. 2017;70(1):155-62. https://doi.org/10.1590/0034-7167-2015-0169

17. Martins MAP, Costa JM, Mambrini JVM, Ribeiro ALP, Benjamin EJ, Brant LCC, et al. Health literacy and warfarin therapy at two anticoagulation clinics in Brazil. Heart. 2017;103(14):1089-95. https://doi.org/10.1136/heartjnl-2016-310699

18. Santos JEM, Brasil VV, Moraes KL, Cordeiro JABL, Oliveira GF, Bernardes CP, et al. Comprehension of the education handout and health literacy of pacemaker users. Rev Bras Enferm. 2017;70(3):633-9. https://doi.org/10.1590/0034-71672016-0336

19. Rodrigues R, Andrade SM, González AD, Birolim MM, Mesas AE. Cross-cultural adaptation and validation of the Newest Vital Sign (NVS) health literacy instrument in general population and highly educated samples of Brazilian adults. Public Health Nutr. 2017;20(11):1907-13. https://doi.org/10.1017/S1368980017000787

20. Souza DP, Silva WRS, Cervinski GC, Santos B, Comarú FA, Trigoso FBM. Desenvolvimento urbano e saúde pública: impactos da construção da UHE de Belo Monte. Desenvolvimento e Meio Ambiente. 2018;46:154-73. https://doi.org/10.5380/dma. v46i0.56040

21. Carvalho GB, Amaral MDB, Herrera JA. A reprodução urbana em Altamira-Pará: uma análise dos reassentamentos urbanos coletivos - 2016. Geografia (Londrina). 2019;28(2):101-21. https://doi.org/10.5433/2447-1747.2019v28n2p101

22. Instituto Brasileiro de Geografia e Estatística. Censo Brasileiro de 2010. Rio de Janeiro: IBGE; 2012. Disponível em: https:// www.ibge.gov.br/cidades-e-estados/pa/altamira.html

23. Altin SV, Finke I, Kautz-Freimuth S, Stock S. The evolution of health literacy assessment tools: a systematic review. BMC Public Health. 2014,14:1207. https://doi.org/10.1186/1471-2458-14-1207

24. Paasche-Orlow MK, Parker RM, Gazmararian JA, Nielsen-Bohlman LT, Rudd RR. The prevalence of limited health literacy. J Gen Intern Med. 2005;20(2):175-84. https://doi.org/10.1111/j.1525-1497.2005.40245.x

25. Sørensen K, Pelikan JM, Röthlin F, Ganahl K, Slonska Z, Doyle G, et al. Health literacy in Europe: comparative results of the European health literacy survey (HLS-EU). Eur J Public Health. 2015;25(6):1053-8. https://doi.org/ 10.1093/eurpub/ckv043

26. Lima MFG, Vasconcelos EMR, Borba AKOT. Instruments used to evaluate functional health literacy in elderly persons with chronic kidney disease: integrative review. Rev Bras Geriatr Gerontol. 2019;22(3):e180198. https://doi.org/10.1590/198122562019022.180198

27. Nielsen-Bohlman L, Panzer AM, Kindig DA. Health literacy: a prescription to end confusion. Washington: The National Academies Press; 2004. https://doi.org/10.17226/10883

28. Ação Educativa. Instituto Paulo Montenegro. Indicador de Alfabetismo Funcional. INAF Brasil 2018. Resultados preliminares. São Paulo: Ação Educativa. Instituto Paulo Montenegro; 2018. Disponível em: https://acaoeducativa.org.br/wp-content/ uploads/2018/08/Inaf2018_Relat\%C3\%B3rio-Resultados-Preliminares_v08Ago2018.pdf

29. Ação Educativa. Instituto Paulo Montenegro. INAF Brasil 2011. Indicador de Alfabetismo Funcional. Principais resultados. São Paulo: Ação Educativa. Instituto Paulo Montenegro; 2011. Disponível em: https://acaoeducativa.org.br/es/wp-content/ uploads/sites/3/2017/04/informe-de-resultados_inaf2011.pdf

30. Ancker JS, Grossman LV, Benda NC. Health literacy 2030: is it time to redefine the term? J Gen Intern Med. 2020;35(8):242730. https://doi.org/10.1007/s11606-019-05472-y

31. van der Heide I, Rademakers J, Schipper M, Droomers M, Sørensen K, Uiters E. Health literacy of Dutch adults: a cross sectional survey. BMC Public Health. 2013;13(1):179. http://dx.doi.org/10.1186/1471-2458-13179. 